\title{
Étude pragmatique de la relation médecin/patient à partir de données orales authentiques
}

\author{
Aline Delsart ${ }^{1 *}$, Emmanuèle Auriac-Slusarczyk ${ }^{2}$ \\ ${ }^{1}$ Doctorante, Université Clermont Auvergne, ACTe, F-63000 Clermont-Ferrand, France \\ ${ }^{2}$ Maitre de Conférences, Université Clermont Auvergne, ACTe, F-63000 Clermont-Ferrand, \\ France.
}

\begin{abstract}
Résumé. L'étude prend appui sur les données orales du corpus DECLICS2016, concernant des consultations entre médecins et patients en C.H.U. Nous proposons une analyse en deux temps. Premièrement, le corpus de six consultations dans divers services sert d'approche exploratoire pour mettre en évidence l'équilibre interactionnel et certains emplois (régulateurs, marqueurs argumentatifs, répétitions, MSC) contrastant les rôles de médecins et patients. Deuxièmement, à partir de cette étude exploratoire, nous étudions une séquence croisant les principes de l'analyse hiérarchique et fonctionnelle et ceux de la logique interlocutoire. L'étude montre que le médecin doit négocier ses intentions de vérification du diagnostic en intégrant l'expérience du patient. Mots clefs : interaction, consultation médicale, corpus oraux, analyse interlocutoire, négociation.
\end{abstract}

\begin{abstract}
Pragmatics study of the doctor-patient relationship through authentical oral data. This study focuses on oral data of DECLICS2016 corpus, centered on doctors-patients health consultations. We propose an analysis into two steps. On one hand, we explore a corpus of six consultations in several health areas of C.H.U to take into account interactional balance and the uses of conversational regulation, argumentative markers to be contrasted between the patient's $v s$ the doctor's roles. Secondly, on the other hand, based on the first exploratory study, is studied a sequence with the tools of hierarchic and functional theory an interlocutory logic principles. Our study shows that doctor have to negotiate his own intention of make diagnostic truth in front of experiential view developed by the patient. Keys words: interaction, health consultation, oral corpora, interlocutory analysis, negotiation.
\end{abstract}

\section{Introduction}

La présente étude porte sur l'analyse pragmatique et interactionnelle de données orales authentiques, recueillies en milieu hospitalier. Notre étude fait partie du projet de recherche DECLICS (Dispositif d'Études Cliniques sur les Corpus Santé), financé par la région Auvergne-Rhône-Alpes. Ce projet, né d'une collaboration pluridisciplinaire entre des chercheurs en Sciences Humaines et Sociales et des acteurs en santé (médecins et thérapeutes), cherche à cerner la qualité de l'écoute en milieu hospitalier pour repenser la prise en charge thérapeutique de patients dans une période de mutation en médecine (AuriacSlusarczyk, 2019). Pour atteindre cet objectif, les chercheurs en SHS (essentiellement linguistes, psychologues, psycholinguistes) ont constitué un corpus (dénommé corpus DECLICS2016) pour disposer de paroles authentiques, recueillies puis retranscrites au sein

*Corresponding author : aline.delsart@uca.fr 
de plusieurs services d'un C.H.U. Bénéficiant de cette ressource notoire, l'intérêt de notre étude porte sur le relevé et la description d'évènements interlocutoires se réalisant en consultation médicale, genre défini (Lacoste, 1993). Nous nous focalisons sur des échanges entre un patient atteint d'une maladie chronique et son médecin spécialiste. Qu'apporte la pragmatique à l'étude de l'entretien médical ? Quel est l'apport des études interactionnelles dans la caractérisation de la relation médecin/patient, notamment au niveau de la négociation, à partir de données orales authentiques ? Telle est notre problématique.

Pour l'instruire, nous nous sommes appuyées sur deux théorisations et méthodologies complémentaires. Premièrement, le modèle hiérarchique et fonctionnel élaboré par Roulet (1981-1985) revisité par Kerbrat-Orecchioni (1990), sert d'appui à la sélection et à l'analyse d'échanges. Secondement, la logique interlocutoire (Trognon, Batt, Rebuschi et Sorsana, 2010 ; Trognon, 1997), sert de cadre via des outils ad hoc qui affinent les extraits hiérarchisés et mettent en exergue les processus sociocognitifs sous-jacents aux échanges. Nous analysons des extraits d'évènements interlocutoires révélateurs du processus de collaboration pour dévoiler ce qu'une consultation médicale engage chez les acteurs impliqués : médecins spécialistes de leur domaine de savoir et patients experts de leur maladie.

\section{Cadre théorique}

Suivant la logique pluridisciplinaire en SHS du projet DECLICS2016, nous situons, dans un premier temps, le cadre contextuel de cette étude, à savoir celui du milieu hospitalier. Dans un second temps, nous définissons, en appui sur la littérature, l'orientation pragmatique de notre étude. Enfin, nous précisons et définissons la notion de négociation qui est abordée de manière centrale dans l'analyse des interactions médicales.

\subsection{Le contexte de soin}

Aborder le fonctionnement de l'interaction entre un patient et un soignant (Vergely et al, 2010), peut se faire en analysant les mécanismes communicationnels en jeu dans ce type d'échanges, dont on sait le besoin d'ajustement entre le savoir médical du médecin et le savoir expérientiel du patient (Dominicé, 2010). Le discours médical a été étudié sous des angles divers, pour exemple au niveau structural (Cosnier, 1993) ou au niveau relationnel (Fournier et Kerzanet, 2007). Il a été démontré qu'il était un genre à part entière (Lacoste, 1993) pouvant être défini et étudié comme une langue de spécialité (Gafaranga et Britten, 2004). Cependant, peut-on approfondir ces études et avancer en quoi la consultation médicale se rapproche et/ou diffère d'une conversation ordinaire, genre lui aussi étudié (KerbratOrecchioni, 1996). L'orientation pragmatique choisie (Trognon, 1988, 1993 ; Trognon \& Brassac, 1992) fait une large place à la dimension proprement interactive, intersubjective et dialogique fondant la dynamique cognitive et relationnelle des échanges. Après d'autres travaux conduits en santé (Batt, Trognon et Vernant, 2003), nous l'appliquons aux conditions hospitalières. La consultation médicale a sa propre spécificité du fait du rôle central joué par le patient quand il est atteint d'une maladie difficilement curable. On sait, dans le cadre des maladies chroniques, que le patient adopte une posture d'expert liée aux expériences singulières de sa maladie (Tourette-Turgis et Thievenaz, 2013 ; Préau et Siméone, 2018). Aussi, par l'analyse interlocutoire du processus de négociation à l'œuvre en consultation (diagnostiquer, régler le traitement, prévoir la suite des symptômes, etc.) on peut mettre en exergue le rapport particulier développé entre l'expertise scientifique et médicale du médecin et l'expertise profane mais expérientielle du patient. 


\subsection{La pragmatique interactionnelle}

La pragmatique, dévoilant les faits interactionnels, s'inscrit dans la lignée de la théorie des actes de langage (Austin, 1962; Searle, 1972). Elle s'intéresse au fonctionnement de l'interaction (Goffman, 1973) entre plusieurs locuteurs, définie minimalement comme « une interaction est délimitée par la rencontre et la séparation de deux interlocuteurs » (KerbratOrecchioni, 2005). L'interaction participe à l'aboutissement d'un travail collaboratif où plusieurs participants organisent mutuellement leurs discours concourant à un résultat $a$ priori commun, partagé. Appréhender et comprendre le fonctionnement d'une interaction gagne alors à se faire sur la base des principes du modèle hiérarchique et fonctionnel proposé par Roulet et ses collaborateurs genevois (Roulet, 1981 ; Roulet et coll. 1985). Pour ces auteurs, le discours repose sur une double structure : linéaire et hiérarchique, ce que Trognon (1993) reprendra en parlant d'ordre des énoncés et d'enchaînement logique. La structure hiérarchique précise en quoi les différentes transactions se coordonnent, se subordonnent ou s'enchâssent. Le modèle genevois décrit, avec un formalisme a priori simple, l'hétérogénéité du discours. Il est souvent présenté (Kerbrat-Orecchioni, 1990) et utilisé (Fiema \& Auriac, 2013) en repérant les cinq rangs de la plus petite à la plus grande unité constituante du discours : 1. l'acte de langage 2. l'intervention ou tour de parole, 3. l'échange, 4. la séquence et 5 . l'interaction.

Décrire les transactions suppose de délimiter les échanges principaux, subordonnés, circonscrire les épisodes. On gagne à faire appel à la logique interlocutoire (Trognon, 1999 ; 2006 ; 2012) qui restitue davantage encore l'intersubjectivité à l'œuvre au cœur de l'enchaînement entre les tours de paroles (TDP) considérés comme l'échelle primordiale des activités langagières. L'école nancéienne parle de la construction de mondes interlocutoires (Trognon, 2006), qui en intégrant la logique modale des mondes de croyances (Martin, 1987, 1990) statue sur ce qui s'élabore malgré un a priori imprévisible par définition.

\subsection{De la notion de négociation à l'emploi des marqueurs discursifs}

Le terme de négociation peut exprimer des notions diverses. Kerbrat-Orecchioni (2005) définit quatre acceptions : 1 . type d'interactions, telles que les négociations diplomatiques ou commerciales; 2. forme interactionnelle où les participants s'engagent à se mettre d'accord/décider à propos d'un objet de nature variable ; 3. principe de structuration, acception de l'école genevoise (Roulet et al., 1985) ; 4. mode de résolution d'un désaccord ou différend, grâce au processus interactionnel entre deux participants. Nous envisagerons la 3 ème acception de Roulet. Nos analyses déroulent le fonctionnement interactionnel d'une consultation médicale puis identifient quelques évènements relevant d'échanges qui construisent proprement cette négociation interactionnelle.

Toute étude du discours s'appuie implicitement ou explicitement sur une sélection de marqueurs susceptibles de révéler une partie des processus cognitifs sous-jacents à leur usage (Culioli, 1971, 1990, 2002) au point qu'une marque d'un locuteur devient pour l'auditeur une « instruction de traitement » (Caron, 1984), ce qui permet aussi à l'analyste d'interpréter, de décoder le sens implicite des marqueurs (Ghiglione, Kekenbosh et Landré, 1995).

On sait que la succession des tours de parole (Kerbrat-Orecchioni, 1990) entraîne l'emploi de ponctuants de la langue, bon, bien, là, tu sais, etc. appartenant à plusieurs catégories grammaticales (Vincent, 1993), ponctuants soit inter-tours, soit intra-tours. Kerbrat Orecchioni $(1992,2007)$ insiste sur l'importance d'un relevé et/ou d'une attention à porter aux régulateurs verbaux, oui, $m h$, d'accord, termes d'adresse, etc., quand les genevois démontrent, après les travaux de Gülich (1970) sur les signaux de structuration, l'importance 
de considérer les marqueurs de structuration de la conversation, MSC, (Auchlin, 1981) pour révéler ce qui se négocie dans une interaction ordinaire. L'emploi de bon, pour exemple, instruit d'un rapport de places (Kerbrat-Orecchioni, 1987) qui négocie par son emploi la position haute d'un des interlocuteurs (Caron-Pargue et Auriac, 1997). L'ensemble de ces marqueurs, régulateurs, structurateurs, ponctuants se caractérisent paradoxalement par « la perte de leur signification lexicale » (Gülich, 1970). Ces marques, en revanche, servent l'articulation des énoncés (Traverso, 1999) et servent nécessairement d'appui à toute étude interlocutoire (Trognon, voir plus haut).

Dans le rapport de place a priori engendré par une consultation médicale, on peut s'attendre à ce que le médecin, sachant, dispose d'une position haute en vertu du savoir médical qu'il possède, mais aussi en raison de son aptitude à mener un raisonnement clinique (Jouquan, Parent et Audétat, 2013 ; Audétat, Laurin et Sanche, 2011). Les marqueurs argumentatifs, mais, donc, quand même, enfin, etc. nommés parfois connecteurs discursifs (Rossari, 1992) sont des indicateurs d'opérations argumentatives sous-jacentes à la prise de parole.

\section{Cadre méthodologique}

Nous présentons les données utilisées pour cette étude, puis développons notre démarche analytique qui s'est déroulée en deux phases. La première, qualifiée d'approche exploratoire, a dégagé des tendances concernant le déroulement global d'une consultation médicale d'un point de vue processuel. La seconde propose l'étude formelle d'évènements interlocutoires, qui, illustrant une séquence singulière, révèlent le cœur du fonctionnement interactionnel au cours d'une consultation médicale, sous forme d'étude de cas.

\subsection{Description des données exploitées}

L'étude exploite des données verbales issues du corpus DECLICS2016, composé de 42 enregistrements audio, soit 30 heures et 8 minutes. Trois types d'échanges composent ce corpus : 1 . des consultations médicales qui s'effectuent entre un patient atteint d'une maladie chronique et un médecin spécialisé ; 2 . des entretiens cliniques entre un patient et un thérapeute ; 3 . des présentations cliniques apparentées aux présentations de Charcot où le patient rencontre le thérapeute face à un auditoire composé de cliniciens et de chercheurs en SHS. Ces échanges (consultation, entretien, présentation) ont été recueillis dans divers services : neurologie, nutrition, maladies infectieuses et médecine interne. Six patients sont suivis : 1 en neurologie, 1 en nutrition et 3 en maladies infectieuses, soit 12 échanges (cf. Delsart et Marqués 2019). Pour un même patient, une consultation avec un médecin spécialiste puis un entretien ou présentation avec un thérapeute sont considérés comme binômes. Nous analysons la séquence de consultation d'un des patients atteint de VIH avec son médecin infectiologue, qui dure 26 minutes et comptabilise 326 tours de paroles pour 4482 mots.

\section{2. Étude exploratoire : équilibre interactionnel, régulation verbale, cohérence discursive et structuration conversationnelle}

Nous avons mené l'approche exploratoire des six binômes du corpus DECLICS2016 en contrastant la compétence communicationnelle des soignants (médecins et thérapeutes réunis) à celle des patients suivis. Nous décrivons l'équilibre interactionnel en pourcentages comparés de tours de parole et de mots employés par chaque partie (soignants/patients), 
calculons la fréquence d'emploi des marqueurs différenciant les rôles des deux interlocuteurs (médecin-patient) parmi la sélection de régulateurs verbaux ( $m h$, voilà, oui , etc., cf AuriacSlusarczyk et al., 2018) et exploitons la description d'emploi de deux marqueurs argumentatifs de cohérence discursive (mais/donc, cf. Delsart \& Marqués, 2019). Une étude portant sur les hétéro-répétitions donne un aperçu complémentaire du processus interactionnel des six consultations médicales étudiées, précisant pour exemple les moments où la répétition exacte de ce qui est dit sert une fonction de ciblage, de précision, de questionnement, etc. (Delsart, 2019). Au total, on dispose d'une répartition générale chronologique des emplois (régulateurs, hétéro-répétition, cohérence) au fil de la consultation (Delsart et Marquès, 2019).

\subsection{Typification d'évènements interlocutoires}

Les évènements interlocutoires sont étudiés au sein d'un échange présenté sous forme d'arborescence hiérarchique et fonctionnelle, au sein d'un tableau qui met en exergue les emplois au fil des tours de paroles des marqueurs de structurations de la conversation. Sont de plus pointés, pour chaque tour de parole (rang 2 du modèle de Roulet et al., 1985), les actes de langage principaux, représentatifs du découpage que nous opérons.

Extrait 1. Cf. Tableau 1 (voir plus bas)

\begin{tabular}{|l|c|c|}
\hline P69a : mais c'est des malaises & Contre-argumentation (mais) & Déclaratif \\
\hline P69b : ouais & Confirmation (ouais) & Expressif \\
\hline $\begin{array}{l}\text { P69c : après énormément } \\
\text { transpiré + pas mal }\end{array}$ & Précision circonstancielle (après) & Assertif \\
\hline
\end{tabular}

\section{Résultats}

Nous illustrons tout d'abord, grâce à l'emploi singulier de bon, en quoi l'enchaînement caractérise un évènement inhérent aux rapports de places entre médecin et patient, présentons les éléments provenant de l'approche exploratoire des six consultations puis proposons l'étude interlocutoire de la séquence du patient échangeant avec le médecin infectiologue.

\subsection{Approche exploratoire des consultations médicales du corpus DECLICS2016}

La négociation médecin/patient sera abordée progressivement. Après repérage de quelques éléments sur les rapports de place, l'emploi des régulateurs verbaux, et focus sur le raisonnement de chaque interlocuteur, le lecteur disposera d'une vue d'ensemble permettant d'approcher la séquence étudiée au plan interlocutoire.

\subsubsection{Effet de dominance dans les rapports médecins-patients}

L'effet de dominance verbale du médecin, placé en position haute, est marqué par l'emploi de bon (cf. Caron-Pargue et Auriac, 1996). Les deux extraits suivants l'illustrent.

Extrait 2. P désigne le patient, $M$ le médecin ; suivi du numéro de tour de parole. Service de maladies infectieuses

P105 : et là quand vous touchez c'est presque sensible 
M107 : bon + ces épisodes de fatigue + la fièvre à trente-neuf c'est quand même pas enfin tout le monde n'a pas de la fièvre à trente-neuf le soir vous voyez

Dans cet extrait, le marqueur bon propose un enchaînement rétroactif du médecin sur des énoncés antérieurs du patient, et se saisit de cette rétroaction pour orienter le cours d'action de la consultation: ici le médecin profite de la situation pour changer de thème (sensibilité/fièvre et conséquences).

Extrait 3. P désigne le patient, M le médecin ; suivi du numéro de tour de parole. Service de maladies infectieuses

P158 : c'est vrai qu'après les fruits c'est bien $++\mathrm{j}$ 'en mange aussi des fruits ++ faut faire gaffe au glyphosate $\{$ rires

M160 : $\{$ rires $\}$ bon + allez + on va faire ce bilan + d'accord ++ vous prenez un euh rendezvous avec moi dès que possible

Dans l'extrait 2, le marqueur bon a pour fonction de clôturer l'échange, renforcé par l'emploi du ponctuant allez utilisé pour embrayer sur la fin de la consultation. Dans ces deux extraits, le médecin se place en position haute, et domine l'orientation thématique.

Peut-on néanmoins en conclure que le médecin négocie l'échange à son profit?

\subsection{2. Équilibre interactionnel et emploi des régulateurs verbaux}

Dans les consultations médicales, l'équilibre interactionnel n'est au profit d'aucune des deux parties : $49 \%$ des tours de paroles correspondent au patient contre $51 \%$ au médecin. En nombre de mots le médecin partage également la dynamique discursive puisque le patient emploie en moyenne 2437 mots contre 1924 pour le médecin.

Les patients utilisent un peu plus de régulateurs verbaux que les soignants, 17 régulateurs en moyenne contre 15 pour le médecin. Le régulateur le plus utilisé est le oui avec 56 emplois par le patient et 31 pour le médecin, le $m h$ restant moins caractéristique du genre consultation ( 85 emplois en moyenne pour les thérapeutes contre 25 pour les médecins).

\subsubsection{Raisonnement argumentatif : les emplois de mais et donc et les hétéro- répétitions}

En consultation médicale, en moyenne le patient utilise 42 emplois de mais pour 39 emplois du médecin. En revanche, les usages de donc sont en moyenne différents selon les locuteurs, le médecin l'utilisant 29 fois contre 12 pour le patient. La cohérence discursive en consultation médicale différencie les rôles des interlocuteurs, ce que l'étude antérieure des héréro-répétitions consignait aussi : si les thérapeutes répètent à l'identique mots ou expressions des patients, pour un total de $45 \%$ des hétéro-répétitions du corpus DECLICS2016, 32\% sont le fait du patient (face au médecin ou au thérapeute) et $23 \%$ pour seulement le médecin. Ces hétéro-répétitions ont des fonctions différentes : $59 \%$ des hétérorépétitions des patients servent à confirmer les propos des soignants, quand $48 \%$ des hétérorépétitions des médecins visent à partager un savoir avec le patient et favoriser l'intercompréhension (cf. Delsart, 2019). Ci-dessous, nous illustrons avec pour exemple, l'emploi de mais, le déroulement temporel de la consultation étudiée dans le service de maladies infectieuses. L'encadré (Figure 1) indique les bornes de la séquence étudiée qui sera présentée plus bas. 


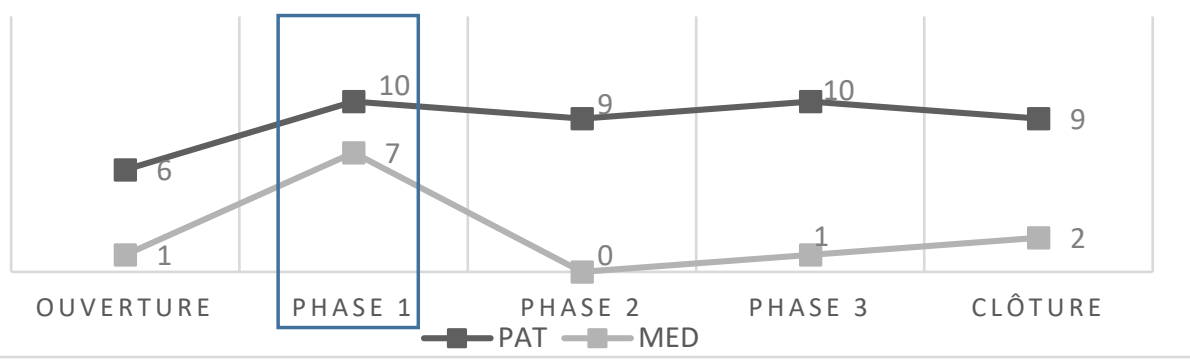

Fig.1.Nombre brut d'occurrences d'emploi de mais dans les différentes phases de la consultation du patient VIH

Au total, l'approche exploratoire, en appui sur plusieurs marqueurs complémentaires, indique une différenciation des places interactionnelles médecin-patient qui oriente sur l'hypothèse d'une négociation interlocutoire particulière en consultation médicale, qui s'écarte de la conversation ordinaire.

\section{2. Étude interlocutoire d'une séquence de négociation patient-médecin}

Présentée sous forme tabulaire (Tableau 1 ci-après), la séquence étudiée révèle les modes d'interventions entrechassées d'une négociation médecin-patient. Les actes de langage principaux (colonne de droite) montrent que les directifs sont systématiquement portés par le médecin (M70, M72, M73, M74, M75) qui scandent ainsi la consultation. Nous représentons sous la forme d'un schéma les incursions répétées du médecin, sous forme d'interrogatoire régulier.

M69 « malaises »

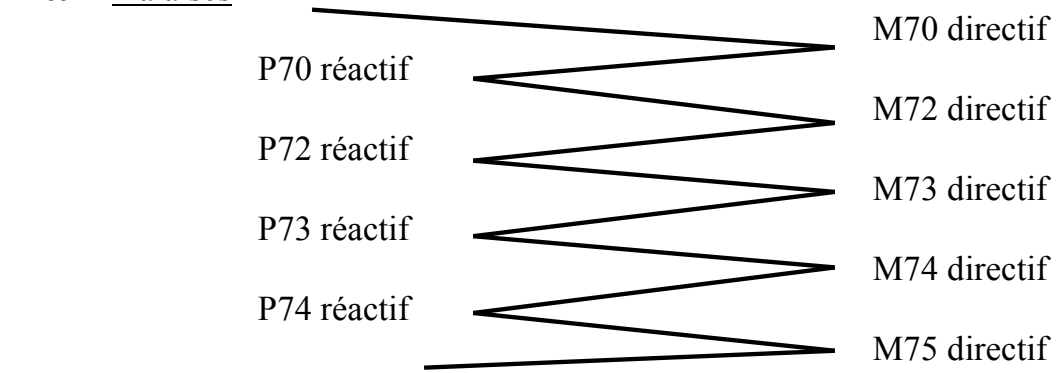

Fig.2. Schéma de la structure d'interrogatoire de la consultation

Les marqueurs verbaux (colonne centrale du Tableau 1 ci-dessous) outillent pour interpréter la colonne vertébrale de la séquence qui s'interprète, au pas à pas, intervention après intervention, mais aussi au sein des interventions, si nécessaire. On peut, selon notre découpage, à chaque pas conversationnel (M69, P69a, P69b, M70, etc.) faire une interprétation de ce qui fait l'objet de la négociation interlocutoire : effet d'insistance, apport de précision, explicitation de symptômes, expression, confirmation, etc. 


\subsubsection{Présentation tabulaire de la séquence}

Tableau 1. Analyse interlocutoire de la consultation en maladies infectieuses

\section{Corpus découpé en interventions au fil de la consultation}

M69: bah surtout que vous avez des douleurs des des des $\underline{\text { malaises }}$

P69a: mais c'est des malaises

P69b : ouais

P69c : après énormément

transpiré + pas mal

M70 : et avec des palpitations

ou pas de palpitations les

malaises \{ton interrogatif\}

P70 : pas spécialement

M71 : pas spécialement

P71 : non pas du tout c'est vraiment euh

M72 : pas de douleur dans la poitrine \{ton interrogatif\}

P72 : je prends chaud

M73 : pas de douleur dans la

poitrine \{ton interrogatif\}

P73a: non +

$\mathrm{P} 73 \mathrm{~b}:$ par contre j'ai eu mal à

la tête ces derniers temps

M74 : pas de douleur pendant la

resp- + pas de gêne pour

respirer \{ton interrogatif\}

P74 : les matins + parfois je suis ge-

M75 : non mais quand vous

avez vos + vos malaises

P75a : ah non non + quand j'ai les malaises non non

P75b : c'est vraiment euh + j' ai ça fait presque un malaise vagal je prends chaud je me mets à suer je deviens un peu livide + je disons que je me mets accroupi ça passe au bout d'une minute une minute trente et après c'est passé +
Marqueurs utilisés en appui de Actes de

l'étude interlocutoire et interprétation du rôle de chaque interventions

langage

principaux

Insistance (surtout)

Déclaratif

Contre-argumentation (mais)

Déclaratif

Confirmation (ouais)

Expressif

Précision circonstancielle (après)

Assertif

Question diagnostic (et avec; ou pas)

Invalidation de la question (pas)

Assertif

Répétition de l'invalidation (pas)

Expressif

Insistance sur l'invalidation (pas

Assertif du tout)

Question diagnostic (pas de)

Directif

Expression d'un symptôme (je)

Déclaratif

Réitération de la question M72 (pas de)

Invalidation de la question (non)

Directif

Suggestion diagnostic (par contre)

Question diagnostic (pas de)

Assertif

Déclaratif

Directif

Précision circonstancielle et

Déclaratif début de déclaration

Contre-argumentation (non mais) et demande de précision circonstancielle (quand)

Invalidation de la vérité et contre-

Directif

(implicite)

argumentation (ah non non)

Explicitation des symptômes et

Déclaratif

des circonstances (je prends

chaud; je me mets à suer; je deviens un peu livide)
Assertif 


\begin{tabular}{|l|l|l|}
\hline $\begin{array}{l}\text { P75c : et ça je sais que c'est } \\
\text { avec la chaleur }\end{array}$ & $\begin{array}{c}\text { Expression de la cause des } \\
\text { symptômes (ça je sais que c'est) }\end{array}$ & Déclaratif \\
\hline M76: d'accord + & $\begin{array}{c}\text { Expression d'accord sur la vérité } \\
\text { (d'accord) }\end{array}$ & Déclaratif \\
\hline
\end{tabular}

Légende : Le médecin est noté $\mathrm{M}$, le patient $\mathrm{P}$ suivi du numéro de tour de parole. Les marqueurs encadrés ou en gras sont repris dans le commentaire. Les marques soulignées indiquent la progression lexicale.

\subsubsection{Commentaire}

Cette séquence débute par la préoccupation du médecin sur des symptômes (M69 « douleurs », « malaises ») adressée au patient ("vous »). Ce dernier accepte d'entrer en conversation sur ce thème en confirmant l'existence du symptôme "malaise » et en en donnant la cause (P69c « énormément transpiré »). Malgré l'explication causale fournie par le patient, le médecin choisit d'approfondir l'énigme du diagnostic en questionnant le patient (directif en M70). On observe alors l'enchaînement répété d'actes directifs et réactifs (cf. schéma Figure 2). Toutefois, à partir de P73b, le patient contre argumente ("par contre ») pour exprimer des symptômes complémentaires aux malaises, et explicite au médecin, à plusieurs reprises, les raisons de ces/ses symptômes, explications qu'il avait amorcé dès sa première intervention (P69c « énormément transpiré », P72 «je prends chaud», P75b «je prends chaud je me mets à suer »). Le patient réussit ainsi à s'immiscer, régulièrement, dans l'interrogatoire (directifs) du médecin pour faire, peu à peu, valoir son savoir expérientiel (P75c « et ça je sais que c'est avec la chaleur »), ancrant de manière répétitive son discours au nom du je (P75b, voir Tableau 1 ci-dessus).

Dans cette séquence, on remarque trois obstacles interactionnels. Premièrement, l'acte directif de M72 échoue puisque le patient ne répond pas à la question, ce qui engendre une réitération de la question par le médecin (M73). Deuxièmement, l'acte déclaratif de P73b échoue puisque le patient évoque un symptôme que le médecin ne reprend pas, continuant la logique de son interrogatoire. Enfin, l'enchaînement M74-P74-M75 rate sa cible (nouveau diagnostic sur la respiration) et oblige le médecin à entrer dans la contre argumentation (« non mais »). Les intentions (illocutoires) de l'un et de l'autre ne s'appliquent pas au même niveau (M se situe au niveau des symptômes alors que P s'en tient aux circonstances, « les matins ", P74 ; « après », P69c ; « ces derniers temps », P73b). En M75, quand le médecin entre dans le monde interlocutoire des circonstances (« quand ») le patient investit alors pleinement ce monde avec un article défini (« quand j’ai les malaises... »).

Qu'est-ce qui est négocié finalement dans cette conversation médicale ? La structure type de la consultation engage un jeu de négociation où médecin et patient se distribuent le pouvoir (places interactionnelles) et le savoir (thème abordé : malaises). Le médecin adopte une posture d'interrogateur, presque d'enquêteur. Il cherche à déterminer la raison des douleurs et malaises du patient et, tel un jeu de piste, il pose des questions au patient pour rassembler un maximum de preuves (les symptômes) pour résoudre cette énigme. Lorsque le patient ne réagit pas dans le sens voulu aux questions, le médecin réitère sa question (M73), reprécise sa demande (M75). Le patient, quant à lui, accepte sa position d'interrogé et suit l'orientation de l'interrogatoire. Cependant, il tente d'expliciter au médecin qu'il connaît les raisons de ses malaises, bornant un monde interlocutoire propre aux circonstances. Plusieurs fois dans cette séquence (P69a, P69c, P72), le patient tente d'expliquer au médecin que le problème se trouve ailleurs (chaleur) et finit par exprimer explicitement ce qui le gêne (P75c). Qui a le mieux négocié sa place ? Le patient-ne détient-il pas la clef de l'énigme posée à ce moment 
de la consultation ? On peut se le demander. Deux mondes interlocutoires s'entrechassent, les échanges matérialisés à gauche par l'arborescence faisant croire à une avancée coopérative, quand le lien thématique maintenu par le patient (chaleur, transpiration, sueur, épisode vagal) occupe le leitmotiv de la consultation.

Monde locutoire du

médecin

Savoir savant / diagnostic
malaises
palpitations
douleurs dans la poitrine
gêne respiratoire
malaises

Monde(s) interlocutoire(s)

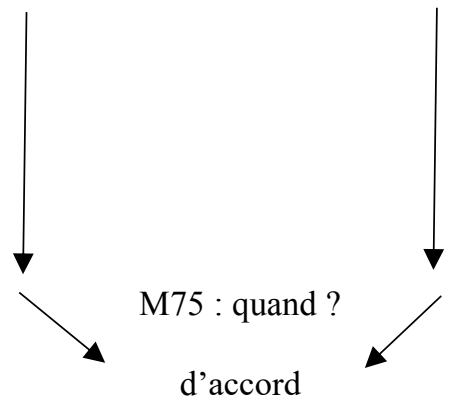

Monde locutoire du

patient

$\underline{\text { Savoir expérientiel / }}$ circonstances transpiration prendre chaud maux de tête

malaise vagal ... prendre chaud ... suer

Fig.3. Articulation des modes locutoires et interlocutoires

Le monde interlocutoire du patient n'est concédé par le médecin qu'en M75, sur un mode proactif contre-argumentatif (non mais) à quoi le patient répond : non non quand... décalant le monde d'enquête du médecin au point de négliger l'enquête diagnostic (malaises, palpitations, douleur de poitrine, gêne respiratoire, malaises) qui revient alors rétroactivement à la question des malaises (M69-M76), mais cette fois en acceptant de parler des circonstances (M75, quand... / malaises). Les deux mondes interlocutoires, du médecin et du patient, ne s'interpénètrent qu'à la fin juste avant que le médecin capitule (M76 : d'accord).

Pour conclure, cette séquence illustre l'expression populaire « dialogue de sourds ». Le médecin s'enferme dans son raisonnement médical. Il cherche coûte que coûte à établir un diagnostic soumettant le patient à un interrogatoire et négligeant les informations données par ce dernier. Le pouvoir du médecin réside dans sa capacité à raisonner, diagnostiquer, décider de traitements ce qui repose sur son savoir de spécialiste en médecine, connaissances des maladies, traitements existants. Le patient, quant à lui, dispose d'un savoir expérientiel lié à son vécu singulier qui entre comme contre-pouvoir dans l'espace de négociation. Ce savoir expérientiel rend ce dernier apte à relier symptômes et circonstances, ce qui lui octroie le pouvoir d'orienter la négociation.

\section{Discussion/Conclusion}

Une consultation est un espace interlocutoire qui se révèle comme un jeu possiblement gagnant-gagnant mais aussi perdant-gagnant, voire perdant-perdant. Que s'est-il réellement passé dans la consultation? La négociation a-t-elle permis que la distribution des pouvoirs augmente le savoir partagé au profit du soin?

L'approche préliminaire du genre consultation médicale (cf. Lacoste, 1993), à partir de l'étude de six consultations, pointe un équilibre interactionnel respecté entre patient et médecin, le patient régulant cependant davantage avec l'affirmative (oui). On pourrait ainsi 
le dire suiveur, ce que l'étude des hétéro-répétitions met aussi en avant (cf. Delsart, 2019). L'analyse des marqueurs de cohésion discursive dévoile en outre que le patient contre argumente le raisonnement clinique (cf. Audétat et al., 2011) élaboré par le médecin (emploi de mais). À ce niveau, on peut dire que la consultation médicale est une négociation située, singulière, à différencier d'une conversation ordinaire (cf. Kerbrat-Orecchioni, 1996).

L'analyse interlocutoire de la séquence, choisie comme cas d'école, retrace finement la structure hiérarchique et fonctionnelle des échanges et dessine les espaces de négociation, sous la forme de mondes interlocutoires construits au pas à pas (cf. Trognon, 1999) par les interlocuteurs. Il apparaît alors clairement, suivant l'interprétation pragmatique de cette séquence, que le médecin se positionne en tant que savant, associant diagnostic et ajustement de traitements à la recherche d'une vérité, ou véridicité via la dialogique (voir Vernant, 2009). L'enchaînement de ses actes directifs suivis des actes réactifs du patient révèle cependant l'enfermement dont fait preuve le médecin où l'activation de son monde interlocutoire transforme la consultation en interrogatoire. Le patient consacre alors ses tours de paroles pour sortir du seul monde locutoire du médecin qui enferme la consultation dans une impasse. N'y a-t-il pas là, mis en évidence, la tension interlocutoire dont fait l'objet toute consultation médicale : négocier entre savoir empirique vs scientifique (patient/médecin) oblige les interlocuteurs à négocier leurs postures interactionnelles. Les mondes locutoires et de croyance respectifs dessinent les lignes de partage entre les pouvoirs de chacun : pouvoir sachant $v s$ expérientiel.

Notre étude ne prétend pas épuiser ce qui se joue dans une négociation de type médical. Elle dévoile des mécanismes précis à partir desquels le médecin peut découvrir l'intérêt d'une analyse linguistique de ses dires. Car de la vérité (du diagnostic, des symptômes) au soin, il y a un pas, et un passage à trouver, que le patient aide à franchir.

Pour compléter l'étude qualitative des faits précis et situés menée, des études quantitatives complémentaires (voir, par exemple, Rendulié et Kanaan-Caillol, 2016) pourraient être envisagées sur les corpus en santé, en explorant systématiquement les emplois d'expressions régulières comme non mais, non non, et/ou les marqueurs temporels, quand. De même, systématiser l'étude des effets d'insistance, sous l'emploi répété du je (P75b), permettrait de vérifier s'il ne traduit pas l'irruption du moi (voir Nowakowska, 2016) du patient dans la scène verbale. Seraient-ce de bons indicateurs pour typifier la négociation médicale d'une manière plus systématique?

\section{Références bibliographiques}

Audétat, M-C, Laurin, S. et Sanche, G. (2011). Aborder le raisonnement clinique du point de vue pédagogique. Un cadre conceptuel pour identifier les problèmes de raisonnement clinique, Pédagogie Médicale, 12(4), 223-229.

Auriac-Slusarczyk, E. (2019). Introduction : Les discours entre soignants et patients. Revue Éducation, Santé, Sociétés, ESASOS, 5(2),7-19.

Auriac-Slusarczyk, E. et al. (2018). Étude pragmatique des discours soignants/soignés en contexte hospitalier. Xème congrès francophone de psychologie de la santé. Metz. 13-15 juin 2018.

Auchlin, A. (1981). MAIS HEU, PIS BON, BEN ALORS VOILA, QUOI! Marqueurs de structuration de la conversation et complétude, Cahiers de Linguistique Française, 2, 141-159.

Austin, J-L. (1962). How to do things with words. Oxford: university press, trad, 1970 Quand dire, c'est faire. Paris : Seuil. 
Batt, M., Trognon, A. et Vernant, D. (2003). Quand l'argument effleure la conviction : analyse interlocutoire d'une croyance dans un entretien de médecine prédictive, Psychologie de l'interaction, 17-18, http://a-trognon.chez-alice.fr/medecine_predictive.pdf.

Caron, J. (1984). Les opérateurs discursifs comme instructions de traitement. Verbum, 7, 149-164.

Caron-Pargue, J. et Auriac, E. (1997). Étude psycholinguistique de la marque conversationnelle 'bon' dans une interaction cognitive. Bernicot, J. Caron-Pargue, J. et Trognon, A. Conversation, Interaction, et fonctionnement cognitif. Nancy : Presses Universitaires de Nancy, 151-185.

Cosnier, J. (1993). Les interactions en milieu soignant. Cosnier, J., Grosjean, M. et Lacoste, M. (dir.). Soins et communication, approches interactionnistes des relations de soins (p.17-32). Lyon: Presses universitaires de Lyon.

Culioli A. (2002). Variations sur la linguistique. Entretien avec Frédéric Fau. Langres : Klincksieck

Culioli, A. (1990). Pour une linguistique de l'énonciation, Opérations et représentations, Tome 1, Paris : Ophrys.

Culioli, A. (1971). A propos d'opérations intervenant dans le traitement formel des langues naturelles, Maths, Sciences Humaines, 9(34), 7-15.

Delsart, A. (2019). Les statuts des locuteurs dévoilés par l'usage des répétitions lexicales en contexte de soin. Journée d'étude Dis-moi ce que tu répètes, je te dirai qui tu es, CeReS, Limoges : 7 juin 2019.

Delsart, A. (à paraître). Les statuts des locuteurs dévoilés par l'usage des hétéro-répétitions locales en contexte de soin. Revue Espaces Linguistiques.

Delsart, A. et Marqués, A. (2019). Analyser les interactions patients-soignants dans une visée formative. Revue Education, Santé, Société, ESASOS, 5(2), 59-83.

Dominicé, P. (2010). Du silence de la souffrance à la parole des patients. Le sujet dans la cité, 1(1), 107-119.

Fiema, G. et Auriac-Slusarczyk, E. (2013). Raisonner en discussion, Cahier du LRL, 5, 230-244.

Fournier, C. et Kerzanet, S. (2007). Communication médecin-malade et éducation du patient, des notions à rapprocher : apports croisés de la littérature, Santé publique, 19(5), 413-425.

Gafaranga, J. et Britten, N. (2004). Formulation in general practice consultations, Text and Talk, 24(2), 147-170.

Ghiglione, R., Kekenbosch, C. et Landré, A. (1995). L'analyse cognitivo-discursive. Grenoble : Presses Universitaires de Grenoble.

Goffman, E. (1973). La mise en scène de la vie quotidienne tome I : la présentation de soi, traduit de l'anglais par Alain Accardo, Paris : Éds. Minuit.

Gülich, E. (1970). Makrosyntax der Gliederungssignale im gesprochenen Französisch, Strucura 2 , München : Fink.

Jouquand, J., Parent, F. et Audétatt, M-C (2013). Des analogies entre le raisonnement médicale et l'évaluation formative, Revue française de linguistique appliquée, XVIII (1), 93-106.

Kerbrat-Orecchioni, C. (2007). Pour une approche interculturelle des termes d'adresse, http://www.mshsud.tv/spip.php?article124

Kerbrat-Orrecchioni, C. (2005). Le discours en interaction. Paris: Éds, A. Colin.

Kerbrat-Orecchioni, C. (1996). La conversation. Paris : Seuil.

Kerbrat-Orrecchioni, C. (1990/1992). Les interactions verbales. Tome I et II. Paris : Éds. A. Colin.

Kerbrat-Orrecchioni, C. (1987). La mise en places. Cosnier, J. et Kerbrat-Orecchioni, C., Décrire la conversation, (p. 319-352). Lyon : Presses Universitaires de Lyon. 
Kerbrat-Orrecchioni, C. (1984). Les négociations conversationnelles, Verbum, 7, 223-243.

Lacoste, M. (1993). Langage et interaction : le cas de la consultation médicale. Cosnier, J., Grosjean, M. et Lacoste, M. (dir.), Soins et communication, approches interactionnistes des relations de soins, (p. 33-61). Lyon : Presses Universitaires de Lyon.

Martin, R. (1990). Pour une approche vériconditionnelle de l'adverbe bien, Langue Française, 88, 8089.

Martin, R. (1987). Langage et croyance. Bruxelles : Mardaga.

Nowakowska, A. (2016). Du fonctionnement dialogique de l'insistance pronominale en français, 5e Congrès Mondial de Linguistique Française, SHS Web of Conferences,27, https://doi.org/10.1051/shsconf/20162702013

Préau, M. et Siméone, A. (2018). De l'expertise scientifique à l'expertise profane. Éditions des archives contemporaines. Lyon: Psychologie du social.

Rendulić, N. et Kanaan-Caillol, L. (2016). Je crois que, je pense que : valeurs et variation dans un corpus oral diachronique, 5e Congrès Mondial de Linguistique Française SHS Web of Conferences, 27, https://doi.org/10.1051/shsconf/20162702014

Rossali, C. (1992). De l'exploitation de quelques connecteurs reformulatifs dans la gestion des articulations discursives, Pratiques, 75, 111-125.

Roulet, E. (1981). Échanges, interventions et actes de langage dans la structure de la conversation, Études de Linguistique Appliquée, 44, 7-39.

Roulet, E., Auchlin, A., Moeschler, J. Schelling, M. et Rubattel, C. (1985). L'articulation du discours en français contemporain. Éditions Peter Lang, collection Sciences pour la communication.

Searle, J-R. (1972). Les actes de langage. Essai de philosophie du langage. Paris : Éds. Hermann.

Tourette-Turgis, C. et Thievenaz, J. (2013). La reconnaissance du "travail" des maladies : un enjeu pour le champ de l'éducation et de la formation. Les sciences de l'éducation pour l'ère nouvelle, 46(4), 68-87.

Traverso, V. (1999). L'analyse des conversations. Paris : Éditions Nathan

Trognon, A. (2012). Connexions dans l'histoire de la logique interlocutoire, Connexions, 98, 83-97.

Trognon, A. (1999). Eléments d'analyse interlocutoire. Gilly, M., Roux, J-P. et Trognon, A. (Dir.), Apprendre dans l'interaction. Nancy \& Aix en Provence : Presses Universitaire de Nancy et Presses de l'Université de Provence, 60-94.

Trognon, A. (1997). Conversation et raisonnement. Bernicot, J., Trognon, A. et Caron-Pargue, J. Conversation, interaction et fonctionnement cognitive (P. 253-283) Nancy : Presses Universitaires de Nancy.

Trognon, A. (1993). La négociation du sens dans l'interaction. Haltié, J-F. Inter-action. Metz: publication de l'université de Metz, 91-120.

Trognon, A. (1988). Comment représenter l'interaction. Gelas, N., Cosnier, J. et Kerbrat-Orecchioni, C., (p. 19-31) Échanges sur la conversation, Lyon : Presses de l’Université de Lyon.

Trognon, A., Batt, M., Rebuschi, M. et Sorsana, C. (2010). Une approche des raisonnements émergents de l'interlocution : la logique interlocutoire, Pratiques, 147/148, 131-154.

Trognon, A., Batt, M., Scharz, B., Perret-Clermont, A-N., Marro, P. (2006). Logique interlocutoire de la résolution en dyade d'un problème d'arithmétique, Psychologie Française, 2(51), 171-187.

Trognon, A. et Kostulski, K. (1997). La logique interlocutoire comme outil d'analyse de l'accomplissement du travail collectif : l'exemple de la négociation commerciale, Psychologie du Travail et des Organisations, 3(1-2), 32-45. 
Vergely, P., Condamines, A., Fabre, C., Josselin-leray, A., Rebeyrolle, J. et Tanguy, L. (2009). Analyse linguistique des interactions patient / médecin. Tardif, F. Actes éducatifs et de soins, entre éthique et gouvernance, Nice : 4-5 juin 2009.

Vernant D. (2009). Discours et vérité. Analyses pragmatique, dialogique et praxéologique de la véridicité. Paris : Vrin.

Vernant, D. (2004). Pour une dialogique de la véridicité, Cahiers de Linguistique Française, 26, $97-$ 111.

Vincent, D. (1993). Les ponctuants de la langue et autres mots du discours. Québec : Éds. Nuit Blanche 